\title{
Traditional Chinese medicine: Pivotal role of the spleen in the metabolism of aristolochic acid $I$ in rats is dependent on oatp2a1
}

\author{
TING XIANG $^{1 *}$, ZHANGBIN YANG $^{1,2^{*}}$, BAOGUO SUN $^{1}$, HAOXUAN LUO $^{1}$, SHIJUN ZHANG $^{1}$,

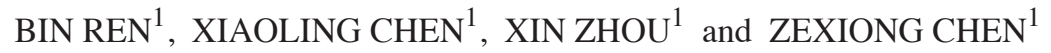 \\ ${ }^{1}$ Department of Traditional Chinese Medicine, First Affiliated Hospital, Sun Yat-Sen University, Guangzhou, \\ Guangdong 510080; ${ }^{2}$ Department of Traditional Chinese Medicine, Fifth Affiliated Hospital, \\ Sun Yat-Sen University, Zhuhai, Guangdong 519000, P.R. China
}

Received August 12,2015; Accepted June 27, 2016

DOI: $10.3892 / \mathrm{mmr} .2016 .5612$

\begin{abstract}
The genotoxicity and cytotoxicity of aristolochic acids is well documented, and the Aristolochiaceae plant family has been widely used in China and India for medical purposes. However, the mechanisms of aristolochic acid I (AAI) in treatment and toxicity remain to be fully elucidated. According to the theory of traditional Chinese medicine (TCM), the spleen is responsible for transportation and transformation, in which a substance is transformed, absorbed and distributed in the body. In the present study, rats were randomized into a blank group without spleen deficiency and a spleen deficiency group to investigate the metabolism of AAI. The results showed that the concentration of AAI was higher in the spleen deficiency group, compared with that of the blank group. To further elucidate this process, the expression of organic anion transporting peptide (oatp) $2 \mathrm{a} 1$ in the rats of the two groups were examined following oral administration of AAI. It was observed that the mRNA level of oatp2a1 in the small intestine of the blank+AAI 60 min group was downregulated, compared with that in the blank group. Compared with the mRNA level of oatp2a1 in the spleen deficiency group, the expression levels in the lung and liver were downregulated in the spleen deficiency+AAI 5 min group, whereas expression levels in the kidney in the spleen deficiency+AAI 60 min group were upregulated. Based on the above results, it was hypothesized that the expression of oatp2a1 may be one of the mechanisms of AAI metabolism in rats. In TCM, the spleen and certain functions of the small
\end{abstract}

Correspondence to: Professor Zexiong Chen or Professor Shijun Zhang, Department of Traditional Chinese Medicine, First Affiliated Hospital, Sun Yat-Sen University, 58 2nd Zhongshan Road, Guangzhou, Guangdong 510080, P.R. China

E-mail: chenzexiongsysu@126.com

E-mail: zhangshijunsysu@126.com

*Contributed equally

Key words: spleen deficiency syndrome, aristolochic acid I, traditional Chinese medicine, organic anion transporting peptide $2 \mathrm{a} 1$ intestine, are important in AAI metabolism, and affect the toxicity of AAI. In addition, the lung, liver and kidney may also be involved in spleen deficiency syndrome in rats.

\section{Introduction}

Aristolochic acids (AAs), a medicinal plant extract of Aristolochia clematitis and Asarum species (1), is widely used for the treatment of snake bites, diarrhoea, rheumatoicd arthritis and gastrointestinal disturbances in Asia (2-4), and are in the homeopathic pharmacopeia in Germany (5). It has been reported in several studies that the ingestion of AA remedies has resulted in AA nephropathy (AAN), which is associated with urothelial malignancy (6-8). AAI, the major component in AAs, is responsible for the nephrotoxicity and the development of urothelial carcinoma $(5,9)$. Additionally, it is well documented that the micromechanism of AAN resulting in renal ischemia combined with impaired regeneration and the apoptosis of proximal tubular epithelial cells, may due to the accumulation of AAI following long-term oral administration $(10,11)$. However, the etiology of AAI-induced renal tubular atrophy and the mechanisms underlying the therapeutic effect of AAI remain to be fully elucidated. As AAI has been documented to be remedial and nephrotoxic, the mechanism of AAI in traditional Chinese medicine (TCM) theory requires investigation.

In TCM theory, food and water is received by the stomach, following which it is transported and transformed by the spleen to enable the substance to be transformed, absorbed and distributed to all areas of the body. The body performs metabolism constantly in association with the spleen, centered through the normal functions of other organs: The nutrients nourish the whole body, whereas the waste products are naturally excreted from the body. The Chinese medicine classic, Jing Mai Bie Lun of Su Wen (Plain Questions), states that, in the first stage, food enters the stomach and moves towards the spleen, which distributes the nutrients upwards to the lungs and directs the waste downwards to the bladder; the nutrients are distributed to the whole body and the waste is excreted from the bladder following metabolism (12). Based on the above concept, the spleen is the predominant organ involved in the transportation and distribution of substances, which 
indicates that the spleen is pivotal in substance metabolism. In TCM, the spleen is comprehensive in structure and function, and is involved in digestion, absorption, energy conversion and immune function $(13)$. Huang et al $(14,15)$ reported that the spleen is also involved in the pharmacokinetics hypothesis, which assumes that the spleen is responsible for the absorption, distribution, metabolism and excretion of drugs. Therefore, the Spleen-Stomach Doctrine (16) was introduced in order to provide a possible approach for the safety assessment of AAI.

The nature and the utilization of AAI indicates that metabolites of AAI are waste products for the body. It is necessary to obtain scientific evidence to verify the traditional theory based on the transportation and distribution of waste products, including AAI. Several previous studies have demonstrated that aquaporins may be associated with water metabolism $(17,18)$; however, few studies have focused on waste metabolites in TCM. It is accepted that organic anion transporting peptides (oatps) can regulate the transportion of endobiotics, xenobiotics and drugs, by which a steady state in the body can be maintained (19-21). A member of a subfamily of oatps, oatp2a1, was first identified in rats in 1995 (22), and is widely expressed in the lung, stomach, intestine, liver and kidney. Of note, these organs are efficient in transporting metabolites and drugs, and they are reported to function in the expression of antilipemic and antineoplastic agents (23-25). The present study hypothesized that these drugs and their metabolites are similar to the waste products of water metabolism. Thus, the expression levels and pattern of oatp2a1 may reflect the transformation and transportation of waste products, including AAI.

The present study was performed to investigate the metabolism of AAI and the expression levels of oatp2a1 in spleen deficiency groups and blank groups without spleen deficiency, in order to confirm the role of the spleen in transformation and transportation. In addition, the present study aimed to preliminarily examine the possible mechanism involved, including other organs, in metabolizing AAI.

\section{Materials and methods}

Animal handling and sampling. A total of 48 male 10 -week-old (250-300 g) Sprague-Dawley (SD) rats, obtained from the Laboratory Animals Central of Sun Yat-Sen University (Guangzhou, China). Rats were housed under a 12-h dark/light cycle, $24 \pm 1^{\circ} \mathrm{C}$ humidity-controlled environment. The rats had free access to food and water ad libitum. The rats were treated in accordance with the National Animal Treatment Guidelines (26). All the animal experiments were conducted in strict accordance with the ethical principles that were approved and supervised by the Ethics Committee of the First Affiliated Hospital of Sun Yat-sen University (approval no. IACUC-2011-0701).

Experimental design. The 48 male SD rats were randomly divided into six groups: A blank group without spleen deficiency (BS), two groups without spleen deficiency administered with $20 \mathrm{mg} / \mathrm{kg}^{-1}$ AAI for $5 \mathrm{~min}$ (BS5) (Delta Pharmaceutical Technology Co., Ltd., Wuhu, China) and 60 min (BS60), a spleen deficiency group (RS), and two groups with spleen deficiency administered with $20 \mathrm{mg} / \mathrm{kg}^{-1}$ AAI for $5 \mathrm{~min}$ (RS5) and $60 \mathrm{~min}$ (RS60). For the establishment of the spleen deficiency model, the rats were injected subcutaneously with $0.5 \mathrm{mg} / \mathrm{kg}^{-1}$ reserpine (Jinyao Amino Acids Co., Ltd., Tianjin, China) (27), with $0.9 \%$ saline used as a control, for 16 consecutive days with an unrestricted diet, activity and sleep. General characteristics of the rats, including the quantity of food and water consumed, body weight, growth, excrement, behavior and activity were closely observed on each day of the experiment.

The experiment was divided into two parts. Experiment 1 involved examination of the metabolism of AAI in the BS and RS groups $(n=6)$. Blood samples $(0.3 \mathrm{ml})$ from the rats were collected at scheduled time points of $5,15,30,45$ and $60 \mathrm{~min}$ following the oral administration of $20 \mathrm{mg} / \mathrm{kg}^{-1}$ AAI. The collected blood samples were immediately centrifuged at $1,006 \mathrm{x} \mathrm{g}$ at $4^{\circ} \mathrm{C}$ for $10 \mathrm{~min}$, and the clear plasma was stored at $-80^{\circ} \mathrm{C}$ for subsequent analysis. Experiment 2 investigated the expression levels of oatp2a1 in tissue samples from six organs (lung, liver, stomach, kidney, small intestine and large intestine). Rats received an overdose of $0.3 \mathrm{ml} / 100 \mathrm{~g}$ weight of $10 \%$ chloral hydrate (Sigma-Aldrich, St. Louis, MO, USA) administered by intraperitoneal injection. Samples ( $5 \mathrm{x} 5 \mathrm{~mm})$ of lung, stomach, liver, spleen, kidney, large intestine and small intestine were removed 5 or 60 min after oral administration of $20 \mathrm{mg} / \mathrm{kg}^{-1}$ AAI. The samples were placed in freezing tubes and preserved within $15 \mathrm{~min}$, following which they were placed in a $-80^{\circ} \mathrm{C}$ refrigerator.

Drugs and reagents. AAI was purchased from Delta Pharmaceutical Technology, Co.Ltd (Wuhu,China) at $20 \mathrm{mg} / \mathrm{pc}$ (cat. no. 20101126) and reserpine was purchased from the First Affiliated Hospital of Guangzhou University of Chinese Medicine (Guangzhou, China) at $1 \mathrm{mg} / \mathrm{pc}$ (cat. no. 101016). Glyceraldehyde 3-phosphate dehydrogenase (GAPDH) mouse monoclonal antibody, poly-L-lysine was purchased from Santa Cruz Biotechnology, Inc. (Santa Cruz, CA, USA). TRIzol was purchased from Invitrogen; Thermo Fisher Scientific, Inc. (Waltham, MA, USA), and the 5X reverse transcription (RT) buffer, $5 X$ quantitative polymerase chain reaction (qPCR) buffer, dNTPs, mMLV and Taq enzyme were purchased from Takara Bio, Inc. (Otsu, Japan). The PVDF membrane was purchased from Bio-Rad Laboratories, Inc. (Hercules, CA, USA). The protein extraction liquid was obtained from the Beyotime Institute of Biotechnology (Shanghai, China). Acrylamide, N-methylene bisacrylamide, Tris alkali and tween-20 were purchased from Sigma-Aldrich. 3,3-diaminobenzidine was purchased from Novolink Biotech Co., Ltd. (Shenzhen, China). The residual immunohistochemical kits were purchased from Golden Bridge Biotechnology Co., Ltd. (Beijing, China). Ammonium persulfate, methanol and glycerol were purchased from Guangzhou Chemical Reagent Factory (Guangzhou, China).

Instruments. The intelligent artificial climate box (BD-PRX) was purchased from Beidi Experimental Instrument Co., Ltd. (Nanjing, China). The 3900 tabletop high flux DNA synthesizer and 7500 Fluorescence Quantitative PCR instrument were purchased from Applied Biosystems; Thermo Fisher Scientific, Inc.). The qualitative PCR instrument, and the vertical electrophoresis and transfer system were purchased from Bio-Rad Laboratories, Inc. The 5417R high-speed refrigerated tabletop centrifuge was purchased from Eppendorf (Hamburg, 
Germany). The MDF-U4084S ultra-low temperature freezer was purchased from Sanyo (Osaka, Japan). The ultrasonic cell disruption equipment was purchased from Touching Technology Co., Ltd. (Shanghai, China). The decolorization table was purchased from Rotomix Company (Shanghai, China). The X-ray photography magazine was purchased from Yijia Biotechnology Co., Ltd. (Guangzhou, China). The RM2235 microtome and ASP300 dehydrator were purchased from Leica (Wetzlar, Germany). The BX41 microscope and BX40F4 digital microcamera were purchased from Olympus Corporation (Tokyo, Japan). The DHG-9123A drying machine was purchased from Jinghong Technology Co., Ltd. (Shanghai, China).

High performance liquid chromatography (HPLC) and pharmacokinetic analysis of AAI. The concentrations of AAI in the above-mentioned treatment groups were measured using HPLC, based on an established method, with AAI used as an internal standard. The mixture, which consisted of a $100-\mu 1$ aliquot of sample in a 2.2-ml eppendorf tube, an internal standard (AAI; $50 \mu \mathrm{g} \mathrm{ml} / 1$ ) in a $50-\mu 1$ aliquot of trichloroacetic acid and a $50-\mu 1$ aliquot of $0.2 \mathrm{M}$ phosphate buffer ( $\mathrm{pH} 7.0$ ), was then extracted with $1 \mathrm{ml}$ diethylether. Subsequently, the organic layer was transferred into a clean eppendorf tube and evaporated under a gentle stream of nitrogen gas at $50^{\circ} \mathrm{C}$. The residue was reconstituted in a $125-\mu 1$ aliquot of the mobile phase, and a 50- $\mu 1$ aliquot was injected directly into a reversed-phase (C8) HPLC column. The mobile phase of phosphate buffer (0.2 $\left.\mathrm{M} \mathrm{KH}_{2} \mathrm{PO}_{4} ; \mathrm{pH} 7.0\right)$ : acetonitrile (77:23; $\mathrm{v} / \mathrm{v}$ ) was run at a flow rate of $1.4 \mathrm{ml} / \mathrm{min}$, with the column effluent monitored using an ultraviolet detector set at $302 \mathrm{~nm}$. The retention times of AA and the internal standard were $\sim 9.8$ and $9.8 \mathrm{~min}$, respectively. The detection limits of the concentrations in the rat plasma and tissue samples were $0.05 \mu \mathrm{g} / \mathrm{ml}$ and $0.31 \mathrm{ng} / \mathrm{g}$, respectively. The coefficients of variation of the concentrations in hte plasma and tissue samples were $<2.81$ and $4.55 \%$, respectively.

Total RNA extraction and RT-qPCR analysis of oatp2al. The extraction of total RNA from the six tissue samples was performed using TRIzol, according to the manufacturer's protocol. The total RNA was reverse-transcribed, and cDNA was synthesized from $4 \mu \mathrm{g}$ of total RNA using primers and Superscript Reverse Transcriptase II (Invitrogen; Thermo Fisher Scientific, Inc.). The final cDNA was used for the subsequent qPCR analysis. The primers were predesigned in the coding DNA sequence of the mRNA sequence of the target gene in GenBank (www.ncbi.nlm.nih.gov/). The primers were designed to amplify DNA fragments, which crossed exon/exon boundaries. The sequence of the forward primer was 5'-CAGCTACTGCTCCCGTCCAT-3' and the sequence of the reverse primer was 5'-CACGCGAAGGACCATCATG-3'. The sequence of the forward primer for the housekeeping gene, GAPDH, was 5'-TGGTCTACATGTTCCAGTATGACT-3' and the sequence of the reverse primer was 5'-CCATTTGATGTT AGCGGGATCTC-3'. The expression was evaluated on a $1 \%$ agarose gel following PCR amplification. The mRNA levels of oatp2a1 were calculated relative to the signal for the target transcript in human tissue samples, which served as a standard. Target mRNA levels were normalized to the internal control gene, GAPDH, according to the following equation: Relative target mRNA level $=2^{-\Delta \Lambda} \mathrm{Cq}$, where ${ }^{\Delta \Delta} \mathrm{Cq}=\left(\mathrm{Cq}_{\text {Target }}-\mathrm{Cq}_{\mathrm{GAPDH}}\right)$ Tissuel $-\left(\mathrm{Cq}_{\text {Target }}-\mathrm{Cq}_{\mathrm{GAPDH}}\right)_{\text {Tissue }}{ }^{2}(28)$.

Western blotanalysis ofoatp2a1. The total protein was extracted form the rat tissues, according to the instructions of the KGP total protein extraction kit. The tissues, which had been cut into sections $(5 \times 5 \mathrm{~mm})$ prior to homogenization, were then extracted by ultracentrifugation at $4^{\circ} \mathrm{C}, 335 \mathrm{x}$ g for $5 \mathrm{~min}$. The concentrations of the proteins were measured using Coomassie brilliant blue staining. In the subsequent step, the separated proteins (20 $\mu \mathrm{l}$ ) underwent 5\% sodium dodecyl sulfate-polyacrylamide gel electrophoresis (SDS-PAGE) and were transferred to polyvinylidene difluoride membranes. The membrane was blocked with 5\% skimmed milk for $2 \mathrm{~h}$. Membranes were then incubated with goat polyclonal anti-Oatp2a1 (cat no. sc-103085; 1:500; Santa Cruz Biotechnology Inc., Dallas, TX, USA) at $25^{\circ} \mathrm{C}$ for $2 \mathrm{~h}$. The membrane was washed with Tris-buffered saline with Tween 206 times ( 5 min per wash). They were then incubated with a horseradish peroxidase (HRP)-conjugated rabbit anti-goat IgG (cat. no. ZB-2306) or HRP-conjugated goat anti-mouse $(\mathrm{H}+\mathrm{L}) \mathrm{IgG}$ (cat. no. ZB-2305) (ZSGB-BIO Biotechnology Co., Ltd., Beijing, China; $1: 5,000)$. at $25^{\circ} \mathrm{C}$ for $1 \mathrm{~h}$. The bands were analyzed using enhanced chemiluminescence (ECL) with the SuperSignal Chemiluminescent HRP Substrates (Thermo Fisher Scientific Inc.). The ECL was detected by films [MXG-1, Kodak (China) Co., Ltd., Shanghai, China]. The results were analyzed by Image J software (Version $1.50 \mathrm{~b}$, National Institutes of Health, Bethesda, MD, USA). GAPDH was detected using mouse monoclonal anti-GAPDH (cat. no. AC002, ABclonal Biotech Co., Ltd., Wobrun, MA, USA), the dilution is 1:5000 utilized as a reference protein to assess the expression levels of the target protein.

Immunohistochemical analysis of oatp2al. The six tissues were fixed in neutral-buffered formalin for $24 \mathrm{~h}$ at $4{ }^{\circ} \mathrm{C}$. Following routine gradient alcohol dehydration, the tissues were sectioned into $6-\mu \mathrm{m}$ sections following being embedded in paraffin. On poly-L-lysine-coated slides, the sections were incubated with dry milk solution for $1 \mathrm{~h}$ at room temperature in order to avoid nonspecific staining. On poly-L-lysine-coated slides, the primary anti-Oatp2a1 antibodies were diluted with dry milk solution to $1: 200$ and incubated at $37^{\circ} \mathrm{C}$ for $1 \mathrm{~h}$. The slides were then incubated with HRP-conjugated rabbit anti-goat $\mathrm{IgG}$ diluted $1: 1,000$ at $37^{\circ} \mathrm{C}$ for $30 \mathrm{~min}$. Finally, images of histological changes were captured using an Olympus microscope, which revealed depositions of brown granular materials in the cytoplasm and nuclei of the cells.

Statistical analysis. The data were analyzed using Statistical Product and Service Solutions (SPSS) 17.0 software (SPSS, Inc., Chicago, IL, USA). The continuous measurement data are expressed as the mean \pm standard deviation and were analyzed using Student's unpaired $t$-test; $\mathrm{P}<0.05$ was considered to indicate a statistically significant difference.

\section{Results}

Comparison of general characteristics. The rats with spleen deficiency treated with reserpine presented with inactivity, 
Table I. Weight, diet and water consumption of rats of the blank group and spleen deficiency groups.

\begin{tabular}{llcr}
\hline Group & Weight $(\mathrm{g})$ & Food consumption $(\mathrm{g})$ & Water consumption $(\mathrm{ml})$ \\
\hline Spleen deficiency $(\mathrm{n}=24)$ & $236.88 \pm 6.62^{\mathrm{a}, \mathrm{b}}$ & $8.14 \pm 1.70^{\mathrm{a}, \mathrm{b}}$ & $9.17 \pm 2.03^{\mathrm{a}, \mathrm{b}}$ \\
Blank $(\mathrm{n}=24)$ & $317.19 \pm 4.68$ & $25.56 \pm 0.98$ & $24.20 \pm 1.11$ \\
\hline
\end{tabular}

Data are expressed as the mean \pm standard deviation. ${ }^{\mathrm{a}} \mathrm{P}<0.05$ and ${ }^{\mathrm{b}} \mathrm{P}<0.01$, compared with the blank group.

somnolence, grouping, loose stools, loss of appetite and anorexia, with lackluster, loose and disorderly fur, which are considered standard characteristics of the spleen deficiency rat model. As shown in Table I, 16 days following spleen deficiency establishment, the weights and the quantities of food and water consumed by the rats in the RS group were significantly lower, compared with those of the rats in the BS group $(\mathrm{P}<0.01)$. This result demonstrated that the spleen deficiency model had been successfully established by reserpine.

AAI pharmacokinetics. As shown in Fig. 1, the concentration-time curves of AAI in the rats following oral administration of a single $20 \mathrm{mg} / \mathrm{kg}^{-1}$ dose of AAI were constructed to examine the intrinsic differences in the metabolism of AAI in the BS and RS groups. The results are presented in the form of separate curves, which showed that the AAI concentrations in the rats of the RS group were higher, compared with those in the BS group between 5 and $60 \mathrm{~min}$, indicating that the metabolic process of AAI in the rats of the $\mathrm{RS}$ group was altered. According to the concentration-time curve of AAI in the RS group, there was a significant increase in the peak concentration, compared with the BS group, with peaking occurring at $45 \min (\mathrm{Cmax} 4.78 \mu \mathrm{g} / \mathrm{ml})$, which was 15 min later than that in the BS group (Cmax $1.42 \mu \mathrm{g} / \mathrm{ml})$, indicating that the metabolism of AAI in RS group was delayed, possibly due to the dysfunction of the spleen.

mRNA expression levels of oatp 2 al. The transcription levels of the transporter, oatp2a1, were detected in the lung, liver, stomach, kidney, small intestine and large intestine tissues of the rats using RT-qPCR analysis. The pairwise comparisons of the results for these tissues were as follows: The mRNA transcription levels of oatp2a1 in the small intestine tissue was downregulated in the BS60 group, compared with the corresponding level in the BS group (Fig. 2A), whereas no difference was observed between the BS5 group and the BS group. However, the mRNA levels of oatp2a1 in the lung and liver tissues were downregulated in the RS5 group, compared with those of the RS group (Fig. 2B), whereas the level in the kidney was upregulated in the RS60 group, compared with that in the RS group (Fig. 2C).

Expression and localization of the oatp2al protein. As shown in Fig. 3, the oatp2a1 protein was generally expressed in the six tissue samples from the rats, including the lung, liver, stomach, kidney, small intestinal and large intestine. Furthermore, as shown in Fig. 4, the patterns of protein distribution observed in the membranes of various tissues samples were similar, including those of the stomach, lung and large intestine. By

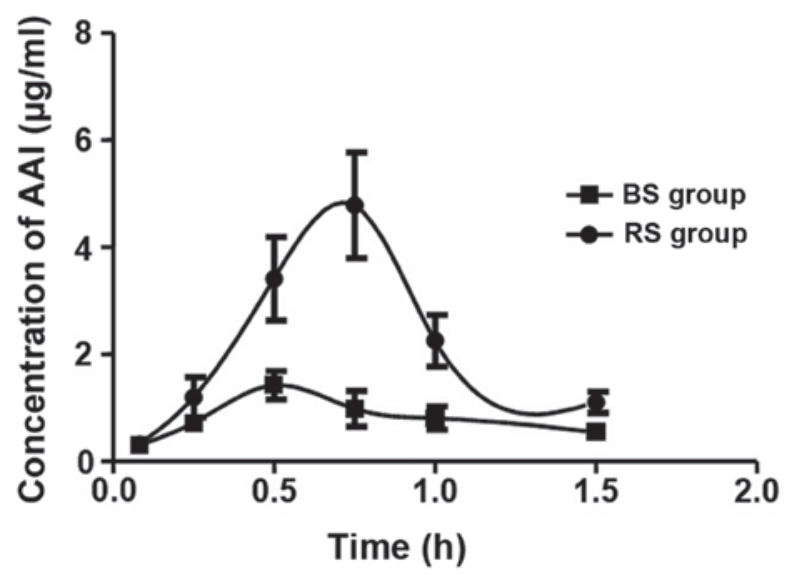

Figure 1. Changes in the concentrations of AAI following oral administration (20 mg kg-1) in rats of the BS and RS groups. Concentrations of AII were measured using high performance liquid chromatographt ( $\mathrm{n}=6$ for each group). The curves show that the AAI concentrations in the rats of the RS group were higher, compared with those of the BS group between 5 and $60 \mathrm{~min}$. Data are expressed as the mean \pm standard deviation. AAI aristolochic acid I; BS, blank (without spleen deficiency); RS, spleen deficiency.

contrast, the expression patterns differed in the liver, kidney and small intestine tissues, in which staining was observed in the membrane and cytoplasm. The immunoreactivity of oatp2a1 was detected in different areas of the tissues, and intense staining was observed in the epithelial cells of the alveoli in the lung and blood sinus, and macrophages in the liver, stomach and renal collecting tubule.

\section{Discussion}

The spleen is a functional unit for substance transformation and transportation in TCM. Due to its importance and complexity, researchers have focused on the properties of the spleen. It is well documented that the spleen is not only an anatomical concept, but is also associated with the digestive system $(29,30)$ in particular, in addition to the immune (31), circulatory (32) and endocrine systems (33). Previously, Huang et al $(14,15)$ suggested that the spleen was involved in the pharmacokinetics hypothesis, which assumes that the spleen is responsible for the absorption, distribution, metabolism and excretion of drugs. However, the role of the spleen has only been demonstrated in absorption (14), and there is no evidence focusing on drug distribution, transportation or excretion. Therefore, the present study aimed to determine whether the spleen affects the entire metabolic process of AAI, which may be associated with the nephrotoxicity of AAI. 
A

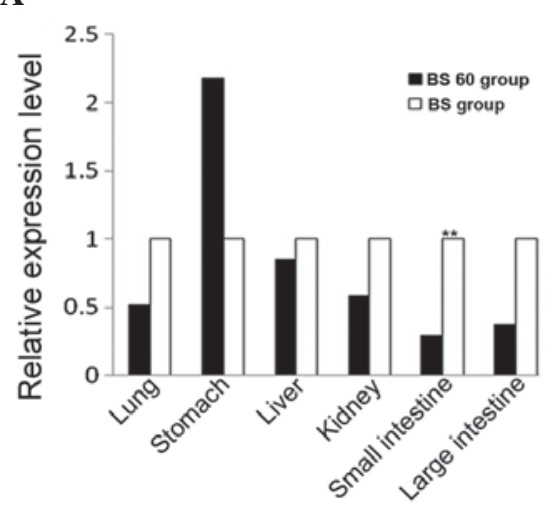

B

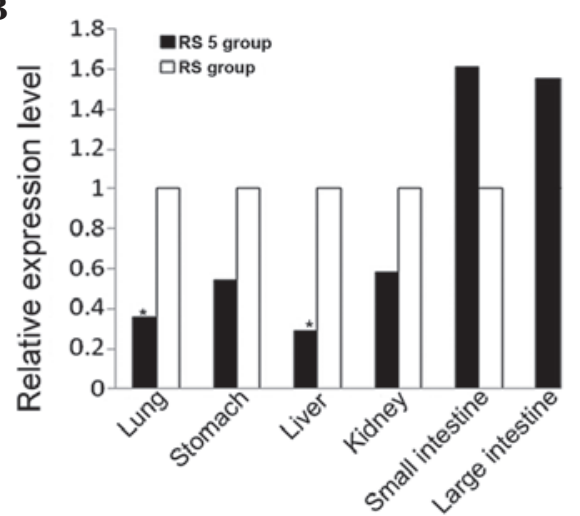

C

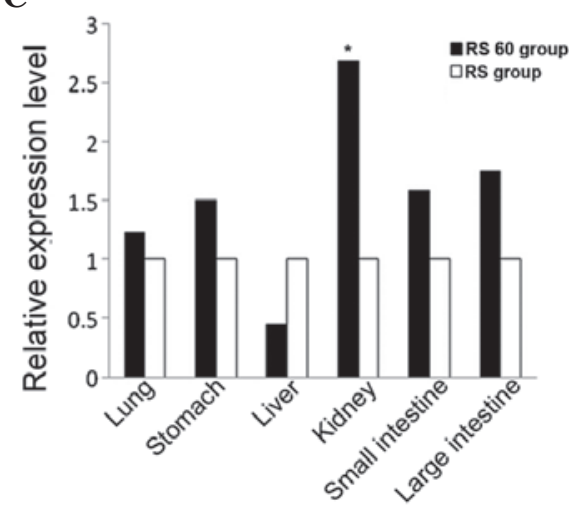

Figure 2. mRNA expression levels of oatp2a1. Reverse transcription-quantitative polymerase chain reaction analysis was performed to determine the mRNA expression levels of oatp2a1 in tissues of the lung, stomach, liver, kidney, small intestine and large intestine. The levels were compared between the (A) BS60 and BS, (B) RS5 and RS, and (C) RS60 and RS groups. All transcript levels are relative to the levels in the respective tissues of the other treatment group (white bars). The values were normalized with those of the housekeeping gene, glyceraldehyde 3-phosphate dehydrogenase. ${ }^{*} \mathrm{P}<0.05$ and ${ }^{* *} \mathrm{P}<0.01$, compared with the same organ in the other tratment group. oatp2a1, organic anion transporting peptide 2a1; BS, blank (without spleen deficiency; RS, spleen deficiency.
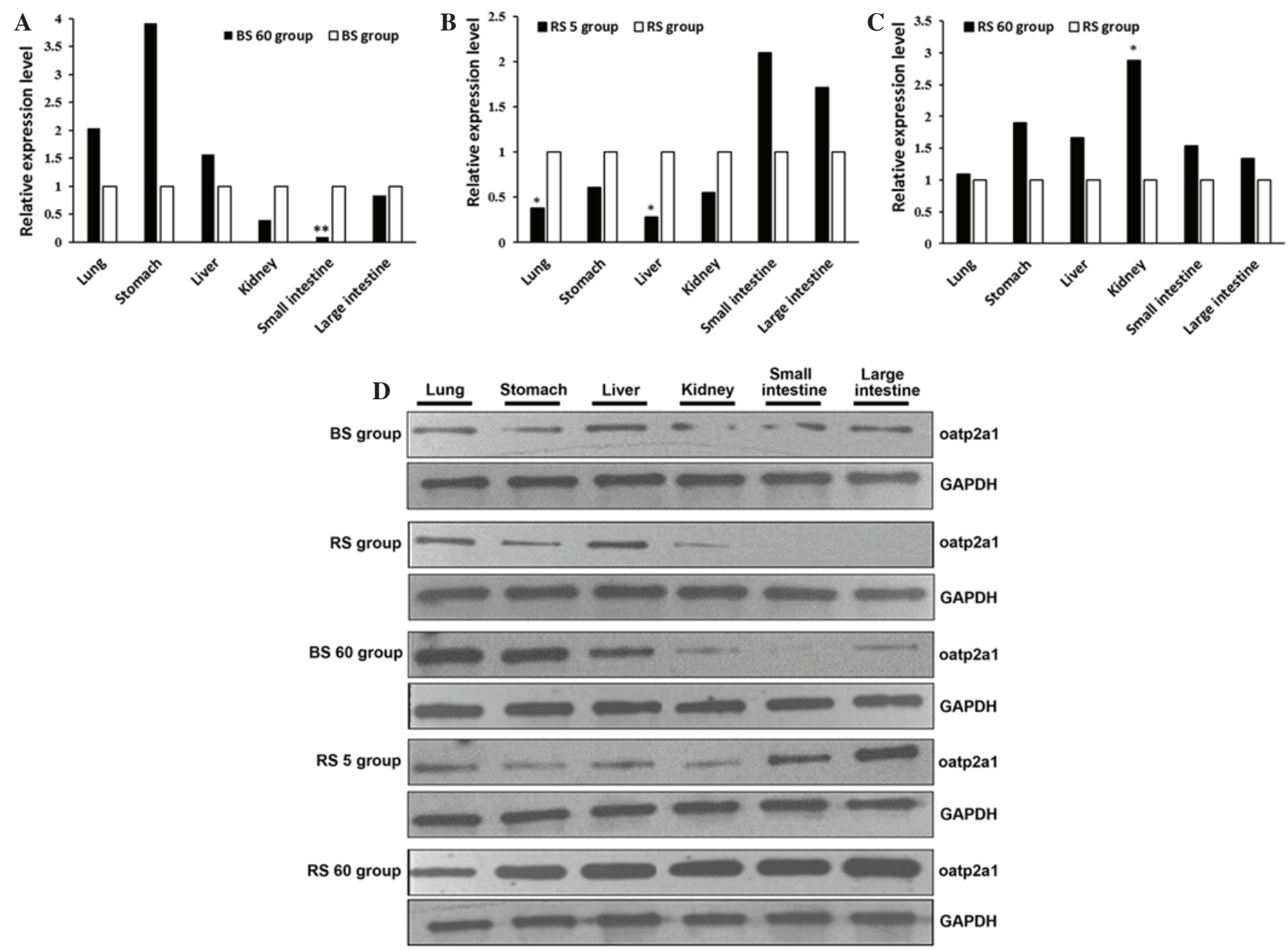

Figure 3. Western blot analysis of the protein expression of oatp2a1 in tissues of the lung, liver, stomach, kidney, small intestine and large intestine of rats. The protein expression of GAPDH was determined as an internal standard. (A) BS60 and BS, (B) RS5 and RS, (C) RS60 and RS groups and (D) western blot results of all groups. " $\mathrm{P}<0.05$ and ${ }^{* *} \mathrm{P}<0.01$, compared with the same organ in the other treatment group. Oatp2a1, organic anion transporting peptide 2a1; AAI aristolochic acid I; BS, blank (without spleen deficiency); RS, spleen deficiency; GAPDH, glyceraldehyde 3-phosphate dehydrogenase.

In the present study, a widely-accepted rat model of spleen deficiency was introduced, which was characterized by pathological changes, including loss of appetite, anorexia, listlessness, somnolence, loose stools and weight loss. As a 

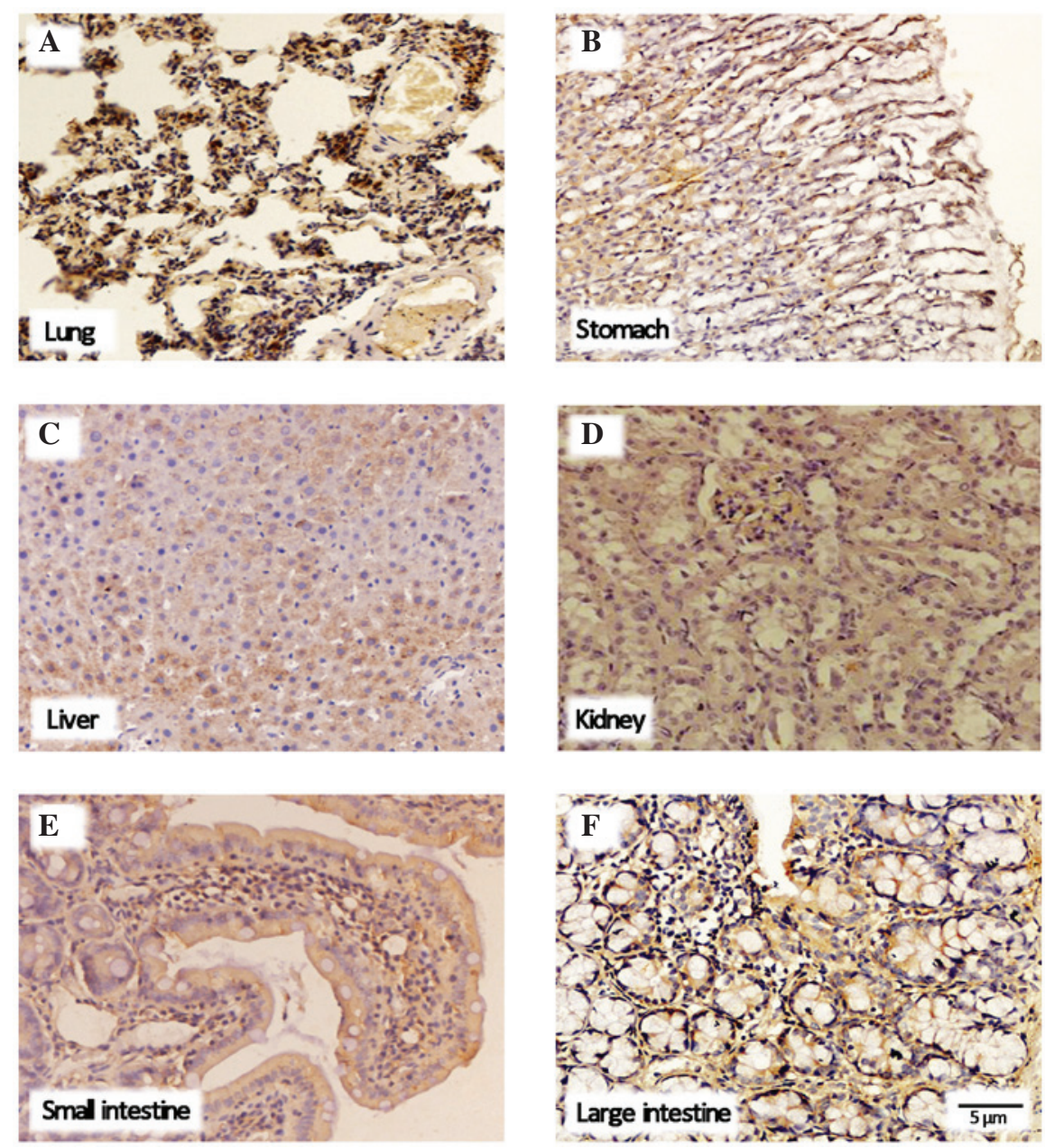

Figure 4. Expression and localization of the organic anion transporting peptide 2a1 protein. Photomicrographs of immunohistochemical stained tissues of the (A) lung, (B) stomach, (C) liver, (D) kidney, (E) small intestine and (F) large intestine 60 min following administration of aristolochic acid I in rats with spleen deficiency. (A-F) Scale bar $=5 \mu \mathrm{m}$.

method for the investigation of spleen deficiency, this animal model has been confirmed to exhibit long-lasting spleen deficiency, which can lead to the dysfunction of digestion and absorption (13).

Orally administered AAI in rats represents an exogenous toxin due to its nephrotoxic, carcinogenic and mutagenic effects $(34,35)$. As shown in Fig. 1, the present study revealed that, when a single dose of $20 \mathrm{mg} \mathrm{kg}^{-1}$ was administrated, the concentration of AAI in the blood of animals in the RS group was higher, compared with that in the BS group for a period of 5-60 min, indicating an abnormally high concentration of AAI with increased toxicity, possibly due to the dysfunction of the spleen. However, the peak concentration of AAI in the spleen deficiency group was observed at $45 \mathrm{~min}$, which was $15 \mathrm{~min}$ later than that in the blank group, following which the concentration decreased rapidly Thus, the absorption, metabolism and excretion of AAI were delayed in the RS group, although the body may accelerate the excretion of AAI by regulating the internal mechanism to maintain homeostasis.

In the present study, the interaction between the heart, spleen, liver, lung and kidney during AAI metabolism was investigated based on the TCM classic principle Jing yue quan shu (Jingyue's Complete Works) (36). It is generally considered that the spleen is pivotal in the transportation and transformation of substances among the other five organs examined in the present study, however, few studies have focused on the transport activities of these organs. Spleen deficiency affected the normal physiological function of other organs, thus the absorption of exogenous organic anions and AAI were altered, as were the metabolism and excretion processes, and the blood concentration of these compounds. In our preliminary study (37), it was demonstrated that the liver is important and associated with the transportation and transformation function of the spleen via the expression level of oatps, however, the expression levels were not compared among these transportation-associated organs.

It has been reported that the absorption, distribution and elimination of endobiotics and xenobiotics in various organs can be regulated by the oatp superfamilies $(19,20)$. Specifically, the function of oatp in the process of drug transportation is well characterized as tissue-specific accumulation and elimination $(38,39)$. In addition, previous studies have found that several members of the oatp family, including oatp2a1, oatp2b1 and oatp4a1, are widely distributed $(37,40)$, and the liver and small intestine are also important in spleen transportation. Among these transporters, oatp2al is expressed at higher levels than the other two oatps. In the present study, analyses of the mRNA and protein expression levels of oatp2a1 
revealed that the changes in mRNA expression levels were observed, not only in the liver and small intestine, but also in the lung, stomach and kidney, and expression was prominent in the blood sinus, macrophage cells of the liver, the stomach, the cytoplasm and membrane of renal tubules and the small intestine. Based on these findings, it was hypothesized that oatp2a1 regulates the transport and excretion of endobiotics and xenobiotics through transmembrane transportation, and may be the mechanism of AAI metabolism, indicated by AAI plasma concentrations.

To clarify the mechanisms and the toxicities of AAI, the present study monitored the process of AAI metabolism, based on the expression of oatp2a1 in six tissue samples. It was demonstrated that the mRNA expression of oatp2a1 remained unchanged in the BS5 group, but was downregulated in the small intestine in BS60 group, compared with the BS group, which is where absorption occurred. These altered absorption levels suggested that the body may decrease the rate of uptake of AAI in the small intestine and increase its excretion to maintain the stability of the environment in rats without spleen deficiency, which may be explained by the hypothesis that the small intestine is important in AAI transport and the maintenance of homeostasis.

In the present study, 5 min following the oral administration of AAI, when the plasma concentration began to change, the expression levels of oatp2a1 were downregulated in the liver and lung tissues of the RS group, but remained unchanged in the BS group. The expression levels of oatp2a1 in the liver cell membranes and the liver macrophages were decreased, predominantly due to the decline in AAI absorption, transportation and phagocytic clearance, respectively. Similarly, the lower expression level in the pulmonary alveolar epithelial cells, which are involved in transportation, indicated a reduction in AAI transportation. Thus, in the state of spleen deficiency, the body reduced the transportation of AAI in the liver and lung, at the beginning of AAI metabolism, which confirmed the importance of the spleen in transporting AAI. At 60 min following the oral administration of AAI, the plasma concentrations were significantly decreased in the two groups, particularly in the RS group. Of note, a novel observation was recorded in the RS group, in that no change in expression was observed in the small intestine, however, the expression level of oatp2a1 was increased in the kidney at the same time. These findings suggested that the kidney excreted more AAI, which may increase the burden on the kidney, and this pathological process may lead to potential renal impairment.

In conclusion, the present study provided experimental evidence of the effects of oatp2a1 on the transformation and transportation of AAI. In addition, the findings suggested the possibility that the function of the spleen involved not only the observation process, but also the distribution and excretion processes of AAI, which may be associated with the function of liver, lung and kidney, and supports the hypothesis that spleen is important among the five organs. Finally, a novel hypothesis was suggested that spleen deficiency accelerates the accumulation of the AAI in the kidney, which may lead to AAI-induced toxicity.

Investigations involving the monitoring of sufficient timepoints are currently in progress to investigate the entire cycle of AAI metabolism. Other approaches, including in vivo labeling are required to examine the mechanism of AAI in TCM.

\section{Acknowledgements}

This study was supported by grants from the National Natural Science Foundation of China (grant nos. 81373500, 81072806 and 81102581) and the Natural Science Foundation of Guangdong Province (grant no. S2012010009277).

\section{References}

1. Cheung TP, Xue C, Leung K, Chan K and Li CG: Aristolochic acids detected in some raw Chinese medicinal herbs and manufactured herbal products-a consequence of inappropriate nomenclature and imprecise labelling? Clin Toxicol (Phila) 44: 371-378, 2006.

2. Wang Y and Chan W: Determination of aristolochic acids by high-performance liquid chromatography with fluorescence detection. J Agric Food Chem 62: 5859-5864, 2014.

3. Schaneberg BT and Khan IA: Analysis of products suspected of containing Aristolochia or Asarum species. J Ethnopharmacol 94: 245-249, 2004.

4. Adeyemi OO, Aigbe FR and Badru OA: The antidiarrhoeal activity of the aqueous root extract of Aristolochia ringens (Vahl.) Aristolochiaceae. Nig Q J Hosp Med 22: 29-33, 2012.

5. Nitzsche D, Melzig MF and Arlt VM: Evaluation of the cytotoxicity and genotoxicity of aristolochic acid I-a component of Aristolochiaceae plant extracts used in homeopathy. Environ Toxicol Pharmacol 35: 325-334, 2013.

6. Wang SM, Lai MN, Chen PC, Pu YS, Lai MK, Hwang JS and Wang JD: Increased upper and lower tract urothelial carcinoma in patients with end-stage renal disease: a nationwide cohort study in Taiwan during 1997-2008. Biomed Res Int 2014: 149750, 2014.

7. Chau W, Ross R, Li JY, Yong TY, Klebe S and Barbara JA: Nephropathy associated with use of a Chinese herbal product containing aristolochic acid. Med J Aust 194: 367-368, 2011.

8. Vanherweghem JL, Depierreux M, Tielemans C, Abramowicz D, Dratwa M, Jadoul M, Richard C, Vandervelde D, Verbeelen D and Vanhaelen-Fastre R: Rapidly progressive interstitial renal fibrosis in young women: Association with slimming regimen including Chinese herbs. Lancet 341: 387-391, 1993.

9. Shibutani S, Dong H, Suzuki N, Ueda S, Miller F and Grollman AP: Selective toxicity of aristolochic acids I and II. Drug Metab Dispos 35: 1217-1222, 2007.

10. Lai MN, Lai JN, Chen PC, Hsieh SC, Hu FC and Wang JD: Risks of kidney failure associated with consumption of herbal products containing Mu Tong or Fangchi: A population-based case-control study. Am J Kidney Dis 55: 507-518, 2010.

11. Schmeiser HH, Nortier JL, Singh R, Gamboa da Costa G, Sennesael J, Cassuto-Viguier E, Ambrosetti D, Rorive S, Pozdzik A,Phillips DH, et al: Exceptionally long-term persistence of DNA adducts formed by carcinogenic aristolochic acid I in renal tissue from patients with aristolochic acid nephropathy. Int J Cancer 135: 502-507, 2014.

12. Lan FL: On English translation of name of sections in 'huangdi neijing suwen' with a comment on the English translation of the section names in two books of English version. Zhongguo Zhong Xi Yi Jie He Za Zhi 24: 265-8, 2004 (In Chinese).

13. Xiong B and Qian H: Effects of Sijunzi decoction and Yupingfeng powder on expression of janus kinase-signal transducer and activator of transcription signal pathway in the brain of spleen-deficiency model rats. J Tradit Chin Med 33: 78-84, 2013.

14. Huang X, Ren P, Wen AD, Wang LL, Zhang L and Gao F: Pharmacokinetics of traditional Chinese syndrome and recipe: A hypothesis and its verification (I). World J Gastroenterol 6: 384-391, 2000.

15. Ren P, Huang X, Li SQ, Xu SY, Wan MH, Zhou YX, Zhou YW and Tang WF: Pharmacokinetic characteristics of ferulic acid in patients with different syndromes of deficiency of spleen qi, stagnation of liver qi and spleen deficiency, and excess of stomach heat. Zhong Xi Yi Jie He Xue Bao 4: 147-151, 2006 (In Chinese). 
16. Wu XN: Current concept of Spleen-Stomach theory and Spleen deficiency syndrome in TCM. World J Gastroenterol. 4: 2-6, 1998

17. Ouyang S, Chen W and Kuang XB: Effects of perindopril on expression of kidney aquaporin-2 and urine aquaporin-2 excretion in chronic heart failure rats. Zhonghua Xin Xue Guan Bing Za Zhi 41 276-281, 2013 (In Chinese).

18. Zhao WX, Gao J, Shi YY and Xu SQ: Effects of different fluid resuscitation regimes on lung injury and expression of pulmonary aquaporin 1 and aquaporin 5 in uncontrolled hemorrhagic shock in rats. Zhongguo Wei Zhong Bing Ji Jiu Yi Xue 21: 282-285, 2009 (In Chinese).

19. Wu LX, Guo CX, Qu Q, Yu J, Chen WQ, Wang G, Fan L, Li Q, Zhang $\mathrm{W}$ and Zhou HH: Effects of natural products on the function of human organic anion transporting polypeptide 1B1. Xenobiotica 42: 339-348, 2012.

20. Csanaky IL, Lu H, Zhang Y, Ogura K, Choudhuri S and Klaassen CD: Organic anion-transporting polypeptide $1 \mathrm{~b} 2$ (Oatplb2) is important for the hepatic uptake of unconjugated bile acids: Studies in Oatp1b2-null mice. Hepatology 53: 272-281, 2011.

21. Meyer zu Schwabedissen HE, Ware JA, Finkelstein D, Chaudhry AS Mansell S, Leon-Ponte M, Strom SC, Zaher H, Schwarz UI, Freeman DJ, et al: Hepatic organic anion transporting polypeptide transporter and thyroid hormone receptor interplay determines cholesterol and glucose homeostasis. Hepatology 54: 644-654, 2011

22. Kanai N, Lu R, Satriano JA, Bao Y, Wolkoff AW and Schuster VL: Identification and characterization of a prostaglandin transporter. Science 268: 866-869, 1995.

23. Mandery K, Bujok K, Schmidt I, Wex T, Treiber G, Malfertheiner P, Rau TT, Amann KU, Brune K, Fromm MF and Glaeser H: Influence of cyclooxygenase inhibitors on the function of the prostaglandin transporter organic anion-transporting polypeptide $2 \mathrm{~A} 1$ expressed in human gastroduodenal mucosa. J Pharmacol Exp Ther 332: 345-351, 2010.

24. Nomura T, Chang HY, Lu R, Hankin J, Murphy RC and Schuster VL: Prostaglandin signaling in the renal collecting duct: Release, reuptake and oxidation in the same cell. J Biol Chem 280: 28424-28429, 2005.

25. Hu ZY: Disposition pathway-dependent approach for predicting organic anion-transporting polypeptide-mediated drug-drug interactions. Clin Pharmacokinet 52: 433-441, 2013.

26. Shirasu T, Koyama H, Miura Y,Hoshina K, Kataoka K and Watanabe T: Nanoparticles effectively target rapamycin delivery to sites of experimental aortic aneurysm in rats. PLoS One 11: e0157813, 2016.

27. Zhao N, Zhang W, Guo Y, Jia H, Zha Q, Liu Z, Xu S and Lu A: Effects on neuroendocrinoimmune network of Lizhong Pill in the reserpine induced rats with spleen deficiency in traditional Chinese medicine. J Ethnopharmacol 133: 454-459, 2011.

28. Corona-Meraz FI, Navarro-Hernández RE, Ruíz-Quezada SL, Madrigal-Ruíz PM, Castro-Albarrán J, Chavarría-Ávila E, Guzmán-Ornelas MO, Gómez-Bañuelos E, Petri MH, Ramírez-Cedano JI, Aguilar-Aldrete ME, Ríos-Ibarra C, Vázquez-Del Mercado M: Inverse relationship of the CMKLR1 relative expression and chemerin serum levels in obesity with dysmetabolic phenotype and insulin resistance. Mediators Inflamm 2016: 3085390, 2016
29. Li ZH, Wang J, Cai RL, Wang YW and Hu JP: Establishment and evaluation of a rat model of ulcerative colitis with syndrome of dampness stagnancy due to spleen deficiency. Zhong Xi Yi Jie He Xue Bao 10: 918-924, 2012 (In Chinese).

30. Li LS, Qu RY, Wang W and Guo H: Significance of changes of gastrointestinal peptides in blood and ileum of experimental spleen deficiency rats. World J Gastroenterol 9: 553-556, 2003.

31. Chen Y, Sun BG, Zhang SJ, Chen ZX, Hardi CF and Xiang T: Observations of TCRV $\beta$ gene expression in rats with dampness syndrome. Evid Based Complement Alternat Med 2014: $373608,2014$.

32. Sponaas AM, Freitas do Rosario AP, Voisine C, Mastelic B, Thompson J, Koernig S, Jarra W, Renia L, Mauduit M, Potocnik AJ and Langhorne J: Migrating monocytes recruited to the spleen play an important role in control of blood stage malaria. Blood 114: 5522-5531, 2009.

33. Grant SJ, Schnyer RN, Chang DH, Fahey P and Bensoussan A: Interrater reliability of chinese medicine diagnosis in people with prediabetes. Evid Based Complement Alternat Med 2013: 710892, 2013.

34. Fuchs TC, Mally A, Wool A, Beiman M and Hewitt P: An exploratory evaluation of the utility of transcriptional and urinary kidney injury biomarkers for the prediction of aristolochic acid-induced renal injury in male rats. Vet Pathol 51: 680-694, 2014.

35. McDaniel LP, Elander ER, Guo X, Chen T, Arlt VM and Mei N: Mutagenicity and DNA adduct formation by aristolochic acid in the spleen of Big Blue ${ }^{\circledR}$ rats. Environ Mol Mutagen 53: 358-368, 2012.

36. Li J and Liu BY: Evaluation of clinical therapeutic effect in Jing yue quan shu (Jingyue's Complete Works). Zhonghua Yi Shi Za Zhi 39: 59-61, 2009 (In Chinese).

37. Pan AZ, Dong XA, Zhang SJ, Xiang T, Chen ZX and Lin YW: Study on mRNA and protein expressions of organic anion transporting polypeptide (oatp2b1) in rats with high fat diet and overstrain induced Pi deficiency syndrome. Zhongguo Zhong Xi Yi Jie He Za Zhi 33: 953-957, 2013 (In Chinese).

38. Takanohashi T, Kubo S, Arisaka H, Shinkai K and Ubukata K: Contribution of organic anion transporting polypeptide (OATP) 1B1 and OATP1B3 to hepatic uptake of nateglinide and the prediction of drug-drug interactions via these transporters. J Pharm Pharmacol 64: 199-206, 2012.

39. Choi MK, Shin HJ, Choi YL, Deng JW, Shin JG and Song IS: Differential effect of genetic variants of $\mathrm{Na}(+)$-taurocholate co-transporting polypeptide (NTCP) and organic anion-transporting polypeptide 1B1 (OATP1B1) on the uptake of HMG-CoA reductase inhibitors. Xenobiotica 41: 24-34, 2011.

40. Dong X, Pan AZ and Sun BG: Organic anion transporting polypeptide (oatp4a1) mRNA and protein expressions in high fat and over-fatigue impairing Pi rats. Zhongguo Zhong Xi Yi Jie He Za Zhi 32: 1223-1226, 2012 (In Chinese). 